was much depressed by the operation, but rallied and recovered. This, I feel sure, would not have been so, had there been failure of immediate union in the stump. To what extent this satisfactory result was attributable to the employment of acupressure instead of ligatures, I cannot tell. I have seen, and not unfrequently, union take place as well when ligatures have been used; and a larger experience is required to enable me to form an estimate of the relative advantages of the new and the old methods. I sincerely hope the morc extended trial of acupressure may prove that Professor Simpson's skill and energy have not only facilitated the performance of operations, but also brought about a real im. provement in the treatment of wounds.

Even if that be so, I do not think that we should rest quite satisfied. The needles may be better than the ligatures; but it would be better still if we could do without either-if we could devise some effectual means of arresting hamorrhage without the necessity for leaving any foreign body in the wound. There seems no impossibility in this; and we will, therefore, hope that it may be accomplished.

\section{CLINICAL REMARKS on}

\section{THE TREATMENT OF EMPYEMA IN CHILDREN.}

BY THOMAS HILLIER, M.D., F.R.C.P.,

Physician to the Hospital for Sick Children, and to the Skin Department of University College Hospital.

PLELRISY is a much commoner disease of children than would be supposed from reading the ordinary treatises on children's diseases. Its presence is often overlooked; the symptoms are referred to "infantile remittent", to " congestion of the brain", or to "disordered liver", and, if it become chronic, to phthisis or atrophy. Pain is either not complained of at all, or else it is referred to the abdomen, or shows itself in a general tenderness of the body; hence the chest is not examined. When a physical examination of the thorax is made, from the frequent absence of friction and the presence of bronchial breathing, with the difficulty of getting nuch information from the voice in young children, the case is often mistaken for inflammation of the lung or tubercular consolidation.

Idiopathic pleurisy is not at all rare in children; pleurisy, secondary to tubercle, to pneumonia, or to renal disease, is equally common, or even more so. Pleuritic effusion in children, even when idiopathic, has a great tendency to become purulent at a very carly period. When pus exists in the pleura, as a general rule, the sooner recoursc is had to para. centesis the better. Even when the effusion is not purulent, if the pleura is much distended, and dyspnoa is increasing, in spite of diuretics and counterirritation, it is unadvisable to postpone the operation. If the emphysema be of very long standing, and the dyspnoe be not great and rather on the decrease, it is better to leave the case to Nature; and the purulent collection will probably excite no irritation, and its fluid portion may be absorbed. In one case of this kind, in a boy five years old, where the discase was of three years' standing, I operated with the patient's chest under water. 'The lung, which had been long collapsed, was atrophied; a communication was established between the bronchi and the pleural carity; this caused decomposition of the contents of the pleura, and the patient dicd in a fortnight of irritative fever.

Of seventeen cases of empycma of which I have notes, in six there were spontaneous openings or pointing of matter requiring operation; of these, five recovered with permanent fistula; the other died, after fourteen months, with necrosis of the sternum and amyloid degeneration of the viscera. In eleven cases, paracentesis was performed; of these, six recovered completely, one had a permanent fistula, and four died. Of the four fatal cases, one was a child only five months old, who died of collapse of the lung; one was twelve months old, and died of pneumonia; one was the child whose case I referred to just now; and another died of septicæmia, whose case will be reported presently.

I believe it is of great importance to prevent the entrance of air into the pleura. I once put the patient into a warm bath, and opened the chest under water. The result in this case has been above mentioned, but was not due to the mode of operation. In some cases, I have evacuated the contents of the chest by means of a long narrow elastic tube whose free end opened under water. Unless air is admitted, and if the lung does not expand very readily, the contents of the cavity are but very partially evacuated. To promote more complete evacuation, the employment of an exhausting syringe, as recommended by Dr. Bowditch (American Fournal of Medical Sciences, vol. xx, Oct. 1850, p. 325; and Gairdner's Clinical Medicine, pp. 380 and 718), is of great service.
Of the last six cases in my wards at the Children's Hospital, in which this syringe has been used, five recovered and only one died. This was a child, twelve months old, who died of pneumonia. He had been previously leeched on his head at a London hospital, and, when admitted, was extremely anxemic and exhausted. These results are probably more favourable than one can usually expect to meet with. Of Bowditch's cases, in twenty-six, the fluid was serous, and twenty-one recovered; in twenty-four, it was purulent-of these, eight recovered, nine were relieved, and seven clied.

The following is a short sketch of my successful cases, in the order of their occurrence. They have all happened since last June; two of them were partially or wholly under the care of my colleague, Dr. Dickinson.

The first was a boy (Armstrong), aged 6 years, who had been suffering from pleurisy of the left side for three weeks. The puncture was made posteriorly between the ninth and tenth ribs, as recommended by Bowditch. Only three ounces of clear yellow fluid were drawn off; it coagulated on cooling. The patient experienced much relief; the wound healed at once, and he made a rapid recovery. In this case, it is very possible that the patient might have got well without operation; but so little risk attends the operation, and so greatly is the chance of recovery diminished by waiting till pus is formed, that, in a similar case, I should again recommend having recourse to the operation, when, at the end of three weeks, dyspnoea and distension of the pleura are increasing in spite of treatment.

The next case was that of a girl (Toynbee), aged 5 years. The disease was of four weeks' standing. There was great dyspnoca and considerable bulging of the side affected (the right). On the first occasion the operation was performed posteriorly in the tenth interspace, and about four ounces of pus drawn off. Considerable relief was experienced. At the end of two days, dyspnoea having returned, and the signs of effusion being better marked in front than behind, I operated in the sixth interspace, a little behind the nipple line, and drew off ten ounces of pus. This gave very great relief. I gave the patient next day a grain and a half of quinine every two hours. Both wounds healed at once. She was again tapped at the end of seven weeks in the fifth interspace, one inch inside the right nipple, and four ounces of non-foetid pus drawn off. The wound healed at once. Four weeks later, she was tapped in the sixth right interspace, an inch and a half outside right nipple. Three ounces of pus were taken, and a permanent cannula in. troduced. There were discharged from day to day quantities of pus, varying from one drachm to ten drachms, for about a month. The quantity of discharge gradually decreased. The tube was removed, and the fistula healed. There remained much flattening under the right clavicle, slight depression of the right shoulder, and slight curvature of spine to the left. Percussion-note over right lung was everywhere deficient in resonance, least so at the apex. Near the nipple, respiratory sounds were almost cavernous; vocal resonance increased. Her general condition was very good. Two months later, the deformity of chest was much less-in fact, almost gone.

Another child (Mingay), a year and a half old, under the care of Dr. Dickinson, was tapped in the ninth and tenth interspaces, below the angle of the scapula, having had pleurisy about seven weeks. One puncture yielded no fluid; the second gave three ounces of bloody pus. She made a good recovery after this, and left the hospital well a week later.

Another case (Ripon), a boy, aged 5 years, had been ill three months. He was tapped four times. The last time the cannula was left in and pus let off every day. A long gum-elastic tube was put in two inches and $a$ half, and pus drawn off. At length nothing could be passed but a piece of wire, and soon the sinus closed. He was four months under treatment; but ultimately left the hospital quite well.

One other case is worth recording. A boy, named Bartlett, aged 4 years, was admitted to the hospital with albuminuria and dropsy after scarlatina. He appeared to be progressing favourably; the albumen de. creasing in amount, and the oedema subsiding; when, sixteen days after admission, he had the symptoms of a cold. Three days later (Nov. loth), his skin was very hot (in axilla, ror $4^{\circ}$ ). He had some dyspncea; and, on examining his chest, the lower half of the left side was found to be dull on percussion; the respiration was somewhat tubular, and the vocal resonance was exaggerated; the heart's apex beat in the line of the left nipple. His pulse was 152, weak; and respirations 58 in the minute. There was general puffiness of the surface. His urine was of a very smoky tint, with a little albumen. He continued much in the same state until the 15th November, when his pulse fell to 128 in the minute, but still very weak; his respirations were 60. His chest presented much the same physical signs. His urine contained a much larger proportion of albumen. The next day, at the base of the left lung posteriorly, a sound resembling redux crepitation was audible. There was now audible for the first time tubular respiration near the angle of the right scapula, 
and for two inches lower down. The oppression of breathing was considerably greater. The urine again contained less albumen. On the 19th November, his pulse varied during the day from 96 to 144; his respirations were 36 . His appetite was better; his bowels were rather loose. The left side of the chest looked fuller than the right, and measured an inch more. The heart's impulse was distinctly felt in the epigastrium. The respiratory sound was tubular over the left lung posteriorly, except near the base, where it was weaker than hitherto. The albumen had almost disappeared from his urine. For the next three days, his general symptoms much improved. The left side of his chest continued as before; the tubular breathing over the base of the right lung had disappeared. On November 26th, he was not so well. There was more dyspnoea; the heart's apex beat in the epigastrium; there was an absolutely dull percussion-note over the left lung, except close to the spine; in front, it extended nearly across the sternum; and the respiration-sounds were everywhere weak. He was tapped with Bowditch's syringe in the seventh interspace in axillary lime. Thirteen ounces and a half of inoffensive pus were drawn off. The next day, he appeared somewhat relieved; his skin acted very freely; his pulse was stronger, 144, and respirations 38. He was sick after the operation. His heart's apex beat in forrth interspace under left nipple. The left side measured only a quarter of an inch more than the right. The dulness over that side was not absolute, and did not extend across the sternum. The respiratory murmur posteriorly was tubular, but weak. He passed about sixteen ounces of urine in the twenty-four hours, specific gravity 1019; on the previous day he had passed but twelve ounces, specific gravity 1018. After this, he improved considerably. There was a tendency to looseness of the bowels. On December 7 th, both sides measured eleven inches and three-quarters below the nipple. The spine was curved considerably to the right. Friction was heard very distinctly over the lower two-thirds of the left lung. On December 1 ith, the left side measured three-quarters of an inch less than the right. Dulness over the left back was less absolute; respiration was weak and tubular ; on deep inspiration, a friction-sound was heard. There was some flattening under the left clavicle. December 18 th. The left side again measured the same as the right; there was great bulging of the chest-wall around and below the left nipple. The heart was pushed more to the right; the dulness and impulse extending to within half an inch of the right nipple. There was a tubular percussion-note below the left clavicle, as low as the third rib, below which the note was absolutely dull, as also in the axilla; respiration was inaudible at this part. Tapping was performed in the fourth left interspace, three-quarters of an inch outside the left nipple, and three ounces of laudable pus escaped. The wound was closed. On December 29th, a little swelling appeared where he was tapped on the 18th. On January 3 rd, it had attained the size of a walnut and was red. It was then punctured. No pus escaped at the time; but, on the 6 th, it began to discharge freely. The pus was not foetid. The swelling looked like a carbuncle. By January i 7 th, the swelling had subsided; the sinus discharged about a drachm of inoffensive pas daily. Much flattening had taken place under the left clavicle. He was now sent to Brigitor. He came back in about two months, with the sinus healed, and without any deformity except a slight flattening under the clavicle. The lung had almost completely recovered itself.

The treatment by drugs was briefly the following. At first, infusion of digitalis, in drachm doses, with acetate of potash and acetate of iron. A few days later, a mixture containing citrate and carbonate of ammonia was given. This was soon followed by a mixture containing two grains of iodide of potassium and four grains of nitrate of potash, with digitalis. On November 23rd, the syrup of the iodide of iron, with bicarbonate of potash, was given. On the $28 \mathrm{th}$, two grains of iodide of potassium were added to the mixture. On December 1st, he was ordered two grains of quinine and one grain of iodide of potassium four times a day. This he contimed till December 29th. From that time until his discharge, he did not take much medicine, except chalk and bismuth occasionally to control his diarrhoea, which recurred from time to time.

The case at one time was extremely unpromising. The occurrence of pnexmonia in both lungs in a patient suffering from scarlatinal dropsy and nephritis, followed by empyema on one side, presented a complica. tion of disease from which recovery could scarcely be anticipated.

The use of Bowditch's syringe enabled us to nearly empty the pleura, and then close the wound without admitting air. Althongh the operation was twice repeated, no foetor resulted.

A case has been recently under my care, which presents an unfavourable contrast with the preceding. The operation was performed before the child came under my notice, with an ordinary trocar and cannula. The mode of death, and the post mortem appearances, are of great interest.

M. A. K., aged two years and a half, had been ill six weeks. Her illness began with vomiting, fever, cough, and pain near the navel. On April 3 rd, she was admitted to the Children's Hospital, looking ex- tremely ill, livid, and unable to lie except on her right side. There were all the signs of a large amount of effusion in the right pleura. A trocar was introduced, and a good deal of pus drawn off. Some air entered. A cannula was put in and closed. The next day, her pulse was 146; respirations 6o. Colour of lips tolerably good. She had passed a restless night. Air entered the right lung moderately well under the clavicle and over the posterior part of the lung. Percussion was dull, except near the spine. She was ordered half a grain of quinine and two minims of dilute sulphuric acid every four hours; milk, beef-tea, and brandy (an ounce and a half in the twenty-four hours). April gth. She had improved a good deal until this afternoon. She now looked very low; lips pale and somewhat livid. Pulse 168, weak; respirations 72. She had just vomited. The bowels were costive. Respiration over the left lung was tolerably free from rhonchi. There was abundant foetid discharge from the wound. April 12th. The discharge was still very fotid. The empyema was washed out with a lotion containing ten grains of carbolic acid to the ounce. April 18th. Fotor was still very great, in spite of injections. She was ordered to use two drachms of tincture of iodine with three drachms of water as an injection, after washing with water. April 22nd. She felt tolerably well, and did not seem to be losing flesh. The discharge was less horribly fotid; watery. The heart's apex-beat in the fifth interspace in the line of the nipple. The right side measured less than the left. Respiration was weak all over the right side. April 3 oth. The discharge was very offensive; not profuse. Iodine injections were continued daily. She took food well. It was determined to consult with the surgeon at his next visit, as to the possibility of making a counter-opening to prevent the accumulation of the offensive fluid in the pleura. She seemed quite in her usual health. May Ist. Whilst the house-surgeon was washing out the empyema, and having filled it as completely as he could with warm water, the child fainted, and ceased to breathe. By the use of artificial respiration and brandy, she revived, but did not regain her consciousness. $A$ few hours later, convulsions set in, chiefly of the right side, and continued with short intermissions for twenty hours, at the end of which she died. The convulsions involved both sides after a few hours, and ceased about an hour before death. For the last hour of life there was regurgitation of a dark brown fluid from the stomach.

On post mortem examination, the arachnoid on the upper surface of the brain was in places milky, with a considerable quantity of fuid in the meshes of the pia mater. Many white opaque spots near the longitudinal fissure. There were numerous dark purple ecchymoses, of the size of pins' heads, in the pia mater, extending in some places into the grey substance. About an ounce of clear fluid in the lateral ventricles. The whole of the brain-substance was abnormally soft; the central portions and parts of the posterior lobe were almost pulpy. In the convexity of the anterior lobe an abscess was found, containing about a drachm of greenish inodorous pus; the abscess was connected with the pia mater at one spot; the pyogenic membrane was rather thick. There was no sign of tubercle or opacity of cerebral membranes at the base. There were no thrombi, or diseased bone. There was no displacement of the heart. The wound was in the fourth right interspace; a quantity of very foetid dirty watery fluid was found in the right pleura. The lung was adherent in front, on the level of the third rib bounding the cavity. This lung was completely collapsed, except anteriorly, and was covered by a tough layer of lymph. It weighed three ounces and a quarter. The left lung weighed two ounces fourteen drachms. The more dependent part of the lower lobe lay in a dirty brown fluid, which was found to have escaped from a longitudinal opening in the oesopha. gus. This part of the lung was stained and mammillated on the surface; there were some ecchymosed spots on the pleura near. The mammillation was due to destruction of the pleura and some of the cellular tissue connecting the lobules. The oesophagus was cleanly dissected out from its connections; and close to the diaphragm at its posterior aspect was an opening, with bevilled edges and no injection around it, measuring an inch by the third of an inch. The stomach contained some food; and was not digested on its surface.

The occurrence of collapse, followed by convulsions, setting in whilst the empyema was being distended with warm water, can scarcely be sup. posed to have been a mere coincidence. The patient had appeared to be going on quite as well as usual up to that time. I am inclined to believe that absorption of the foetid fluid in the pleura was promoted by its dilution with warm water and pressure, and this caused the symptoms. The petechiæ on the surface of the brain, and the general softening of its substance, were no doubt due to septicæmia. The abscess in the brain was no doubt pyæmic in its origin; it probably existed before the convulsions appeared. It is a remarkable feature in the case, that the lungs and liver showed no signs of secondary deposits or suppuration, whilst the brain was so much involved. There were some petechixe on the pleura; but neither apoplexy nor exudation in the lungs. 
The erosion of the œsophagus, and its separation from surrounding parts by the destruction of cellular tissue, as well as the erosion of the right lung in its most dependent part, were interesting, and must no doubt be ascribed to the gastric fluid which collected in the gullet just before death. It was certainly unwise to force water into the pleural cavity which previously contained fotid fluid; it was done by the housesurgeon through his having misunderstood his instructions. The admission of air into the pleura at the time of the operation of paracentesis was not contemplated, and would have been avoided; but from the great restlessness of the child this plan did not succeed. The best way to prevent the admission of air is at once to puncture the skin and other tissues with the cannula, then to draw off as much fluid or matter as possible with an exhausting syringe, and at once to close the opening, which usually unites by first intention. If it is necessary, the operation may be repeated from time to time.

Conclusions.-Paracentesis thoracis is not a dangerous operation in children; if performed early, the chances of a favourable result are very great. The cavity should be evacuated as completely as possible without the admission of air, and then closed. If the contents of the cavity become foetid, a counter-opening should be made, and a drainagetube introduced, as recommended by Chassaignac and Dr. Goodfellow. Injections of iodine tend to diminish fœetor, but have no effect in promoting the closure of the cavity. Change of air, nutritious food, and tonics, are the main agents in favouring recovery. Very great deformity of the chest from contraction after pleurisy in children may be completely removed if the lung have not been too long disabled by compression.

\section{ABSTRACT OF \\ REPORTS AND DISCUSSIONS ON CHOLERA IN DUBLIN : WITH COMMENTS.}

\section{BY GEORGE JOHNSON, M.D.,}

Physician to King's College Hospital; Prcfessor of Medicine in King's College.

THE last number of the Dublin Quarterly Fournal of Medical Science contains two long articles on Cholera. There is first a report by Drs. Hayden and Cruise, giving the results of their experience at the Mater Misericordiæ Hospital ; and secondly, under the heading "Transactions of the Medical Society of the King and Queen's College of Physicians", there is an account of three special meetings which were held to receive and consider reports from the various hospitals in which cases of cholera had been treated. I have carefully read these papers, with a view to extract from them any facts which may tend to increase our knowledge of the disease.

An interesting observation is recorded by Drs. Hayden and Cruise. In several cases of collapse, the second sound of the heart was absent. The failure of the second sound occurred first in the aorta, and, after an interval of a few hours, but varying with the gravity of the case, in the pulmonary artery also. For some time longer, the first sound continued to be audible, ultimately failing, and, in a few instances, several hours before death. The authors contrast this phenomenon with the occasional abolition of the first sound in typhus, the result of softening and muscular debility of the ventricles. They affirm that, in cholera, the walls of the ventricles, especially the left, have been found firmly contracted and unyielding in nearly every case in which an examination of the body has been made within a few hours after death. Reference is made to Skoda's observation, confirmed as it has been by others, that obstruction at the mitral orifice intensifies the second sound in the pulmonary artery, while that in the aorta is in a corresponding degree enfeebled. They remark: "That in the collapse of cholera there is obstruction of the venous circulation through the lungs, the engorged state of the pulmonary artery and the right side of the heart, found after death, clearly shows." These interesting observations of Drs. Hayden and Cruise are an addition to the evidence which before existed that during the collapse of cholera there is an impeded circulation through the lungs; so that, while the pulmonary artery is distended with blood which closes the valves with an audible sound, the aorta is comparatively empty, there is no tension of its walls, and its valves close inaudibly.

Dr. Henry Kennedy observed that, in cases of extreme collapse, puerile respiration was often heard, and was a sure precursor of death. Dr. Stokes commented upon this, and compared it with the occurrence of puerile respiration, either constant or paroxysmal, in cases of fatty degeneration of the right side of the heart. It is not difficult to explain the occurrence of puerile respiration in extreme cholera collapse. It is a result of the rapid and forcible respiratory efforts. What, then, is the cause of this hurried and forcible breathing? Probably the extreme scantiness of the blood-stream through the pulmonary capillaries. Hurried and difficult breathing may result from an excess or from a great deficiency of blood in the pulmonary capillaries. It occurs in all cases of capillary engorgementfor instance, the pulmonary engorgement, which is so commonly associated with the consecutive fever of cholera. On the other hand, it occurs in cases of profuse hæmorrhage, embolism of the pulmonary artery, extreme cholera collapse, and, as Dr. Stokes observed, in cases of fatty degeneration and feebleness of the right side of the heart. Now, that which is common to all these latter cases is a defective blood. supply to the pulmonary capillaries. The causes of this deficiency of blood are different in each class of cases. In cases of hæmorrhage, the blood is absolutely gone from the body; in embolism of the pulmonary artery, the blood is stopped by a mechanical plug; in cholera collapse, the vital contraction of the minute pulmonary arteries lessens the cur. rent; while, in cases of degeneration of the right side of the heart, the pulmonary circulation fails because the propelling power of the heart is defective. In all these cases of defective capillary circulation through the lungs, the respiration is rapid, forcible, and therefore puerile on auscultation. The fact that, in cases of extreme cholera collapse, the respiration is puerile, is in itself evidence that in that condition there is not capillary engorgement of the lungs. In all cases of dyspnoea with capillary engorgement, the natural respiratory murmur is feeble, and associated with fine crepitation. The natural sound is feeble, because capillary engorgement interferes with the free expansion of the lung; and it is mixed with crepitating sounds, because capillary engorgement is quickly succeeded by exudation of liquid into the air-passages. Puerile respiration, then, in the collapse stage of cholera, is a sign of unfavourable import, because it is associated with such extreme obstruction to the pulmonary circulation as places life in imminent peril.

The records of the post mortem examinations are not given with suffi. cient detail to be of much value. The lungs were pale in front, congested at the back. In no case is the weight of the lungs stated, or the period after death at which the examination was made. The right cavities of the heart were full. "The left chambers were empty and contracted; the left ventricle much diminished in capacity, firm, and thickened, manifestly by strong contraction of its walls." It is evident that such appearances are inconsistent with the theory that the circula. tion in cholera fails from want of contractile power in the heart.

The question of the portability and the contagiousness of cholera was very fully discussed. The facts and the arguments greatly prepon. derated on the side of the contagionists, notwithstanding the acknow. ledged difficulty of accounting for such an outbreak as occurred amongst the isolated inmates of Mountjoy Prison.

With regard to the important subject of treatment, it appears to me that there was too much of active interference, too much of mere druggiving, and too little regard to the natural history of the disease. In a large proportion of cases, calomel was given in frequertly repeated doses, and, it is said, "with good results"; but there is nothing to'shew in what way it acted beneficially, if it did so at all. In most cases, alcoholic stimulants were given during collapse; but there is not a par. ticle of evidence that they did good. On the contrary, in some in. stances the mortality was very high under this treatment. Thus, in the House of Industry Hospital, out of forty cases treated by stimulants, there were only thirteen recoveries.

Sir D. Corrigan's button-shaped cautery, heated by boiling water, and applied over the vagus and on either side of the spine, it is said, "would arouse the patient greatly for the time, though he frequently relapsed into his former condition." Is it in the slightest degree pro. bable that any real benefit could ever result from such torture?

Drs. Hayden and Cruise state that, in a few cases, intermittence of the heart and radial pulse was noted in advanced collapse; and "in one of these recovery took place under treatment with large doses of quinine:" Are we then to suppose that the quinine, by virtue of its antiperiodic power, had some specific influence on the intermitting pulse? Again, Drs. Hayden and Cruise state that "the premonitory diarrhoea of cholera is not amenable to the treatment successfully em. ployed in ordinary diarrhœe ; it requires a stimulant and in some degree a peculiar treatment. Chalk mixture, with opium, catechu, etc., which promptly arrests simple diarrhoa, has not usually an equal measure of success in that which precedes cholera, whilst sulphuric acid is all but invariably effectual in checking it." I am quite sure that choleraic diarrhœe treated by sulphuric acid, as a rule, ceases much sooner than when treated by chalk and opium. I believe, too, that a few doses of carbonate of soda in mint-water and even coloured water, without any active drug, are as successful as sulphuric acid, and much more success. ful than opium; and I believe that the reason of this greater success is 